\title{
Spin-polarized tunneling through randomly transparent magnetic junctions: Reentrant magnetoresistance approaching the Jullière limit
}

\author{
Grigory Tkachov ${ }^{1}$ and Klaus Richter ${ }^{2}$ \\ ${ }^{1}$ Max Planck Institute for the Physics of Complex Systems, 01187 Dresden, Germany \\ ${ }^{2}$ Institute for Theoretical Physics, Regensburg University, 93040 Regensburg, Germany \\ (Received 24 March 2008; revised manuscript received 28 April 2008; published 23 May 2008)
}

\begin{abstract}
Electron conductance in planar magnetic tunnel junctions with long-range barrier disorder is studied within Glauber-eikonal approximation enabling exact disorder ensemble averaging by means of the HoltsmarkMarkov method. This allows us to address a hitherto unexplored regime of the tunneling magnetoresistance effect characterized by the crossover from $\boldsymbol{k}_{\|}$conserving to random tunneling ( $\boldsymbol{k}_{\|}$is the in-plane wave vector) as a function of the defect concentration. We demonstrate that such a crossover results in a reentrant magnetoresistance: It goes through a pronounced minimum before reaching disorder- and geometry-independent Jullière's value at high defect concentrations.
\end{abstract}

DOI: 10.1103/PhysRevB.77.184427

PACS number(s): 72.25.-b, 85.75.-d

\section{INTRODUCTION}

Magnetic tunnel junctions with controllable relative orientation of the magnetization in the leads ${ }^{1-3}$ are in the focus of current researches motivated by their promising application potential, as well as general interest in spin-dependent phenomena in complex condensed matter systems. ${ }^{4}$ In particular, among various theoretical studies of spin-polarized transport, a large body of work has aimed at developing adequate models for the tunneling magnetoresistance in singleparticle approximation, ${ }^{5-12}$ by accounting for many-body effects due to electron-magnon interactions in normal ${ }^{13-17}$ and superconducting ${ }^{18,19}$ states, and the influence of disorder. ${ }^{20-23}$

The subject of the present study is the tunneling magnetoresistance (TMR) effect originating from the dependence of the tunneling current on the relative orientation of the magnetizations in two electrodes separated by a thin insulating layer. ${ }^{1-3}$ Usually, the tunnel structure is designed in such a way that in a zero external magnetic field, the magnetic moments are antiparallel (AP) to each other and change to the parallel $(\mathrm{P})$ configuration upon application of a weak magnetic field $B$. If $R(0)$ and $R(B)$ are the resistances in the $\mathrm{AP}$ and $\mathrm{P}$ configurations, respectively, the TMR ratio can be defined as $\mathrm{TMR} \equiv[R(0)-R(B)] / R(0)$ or as $\mathrm{TMR}=\left(G^{\mathrm{P}}\right.$ $\left.-G^{\mathrm{AP}}\right) / G^{\mathrm{P}}$ in terms of the corresponding conductances $G^{\mathrm{AP}}$ $=R^{-1}(0)$ and $G^{\mathrm{P}}=R^{-1}(B)$.

As the effect stems from the exchange interaction, it is not surprising that spin-dependent scattering is often seen as the main obstacle for achieving higher TMR ratios. ${ }^{13-17,24}$ Less obvious is that spin-independent elastic scattering can affect the TMR as well, ${ }^{20-23}$ in particular, in the presence of structural disorder in the insulating barrier. This can be interpreted in terms of disorder-induced mixing of conducting channels with different wave vectors $\boldsymbol{k}_{\|}$in the junction plane occurring independently for the two spin species. On the other hand, according to Jullière's conjecture, ${ }^{25}$ an increase in the amount of barrier disorder should eventually lead to a completely random $\boldsymbol{k}_{\|}$transfer, with the TMR ratio depending only on electron spin polarizations in the magnetic leads. The questions of how the crossover between the $\boldsymbol{k}_{\|}$-conserving and Jullière regimes actually occurs and which of them is more favorable for achieving a higher TMR are the facets of a challenging problem currently under investigation. Although some aspects of this problem have been addressed by numerical techniques, ${ }^{20-23}$ we feel that there is an apparent lack of analytical work aimed at proving Jullière's conjecture from the general standpoint of statistical theory of quantum transport within a model-based approach.

In the present work, we propose an analytically solvable statistical model for spin-polarized tunneling describing the full disorder-driven crossover from the $\boldsymbol{k}_{\|}$-conserving to random tunneling regime. The model assumes nonresonant long-range defects in the barrier and works in the thermodynamic limit where the crossover is controlled by the defect concentration $n$. The scenario of the crossover appears quite unusual, as depicted in Fig. 1: First, decreasing with $n$ at low concentrations, the TMR can eventually recover that of an ideal junction $\mathrm{TMR}_{0}$ or even exceed it, approaching an $n$-independent value for large defect concentrations. As we demonstrate later, this value corresponds to Jullière's TMR for the junctions addressed here. To our knowledge, such a reentrant TMR effect has not been studied previously. This finding could potentially be used for defect engineering of magnetic tunnel junctions.

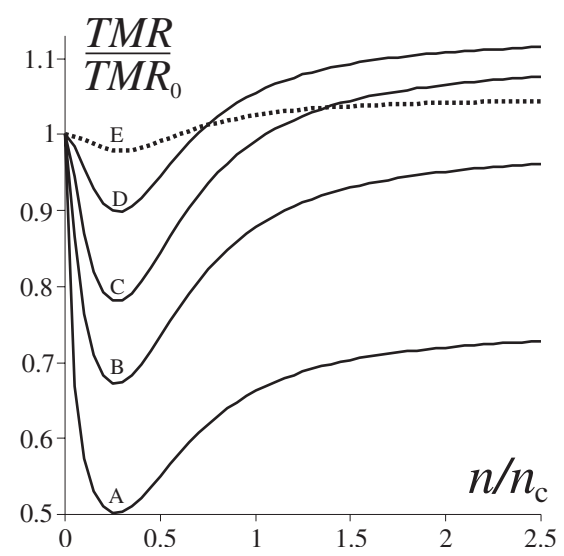

FIG. 1. TMR vs defect concentration for different values of spin polarization: (a) $P=0.01$, (b) $P=0.05$, (c) $P=0.1$, (d) $P=0.2$, and (e) $P=0.4$. $\mathrm{TMR}_{0}$ corresponds to a defect-free junction. The characteristic concentration $n_{c}$ is defined in text [see Eq. (36)]. 


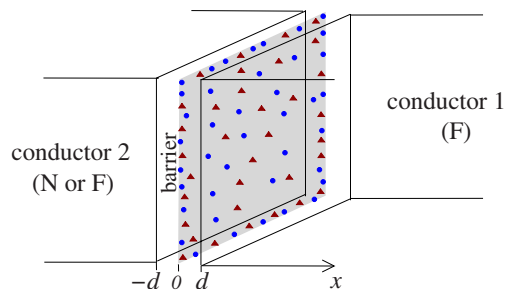

FIG. 2. (Color online) Lateral tunnel junction with an insulating layer of thickness $2 d$ containing two types of defects, schematically shown as circles and triangles, producing a spacial barrier fluctuation described by Eq. (1). System 1 is a ferromagnet, while System 2 can be either nonmagnetic or ferromagnetic.

We also find that the lower the electron spin polarization, the stronger is the effect of the long-range disorder. This, to some extent, is in line with the experimental observation of a rather weak TMR effect in semiconductor/nonepitaxial iron tunnel junctions ${ }^{26,27}$ where one may expect long-range electrostatic disorder in the semiconductor barrier. A quantitative comparison with the experimental data is unfortunately hindered by approximations we have to resort to in order to get an analytically tractable theory.

The subsequent sections give a complete account of our theoretical approach: Section II describes the model for randomly transparent barriers and the main approximations used. In Sec. III, we employ the Holtsmark-Markov averaging procedure to calculate the spin-dependent junction conductance and Sec. IV contains the results and final discussion.

\section{EIKONAL APPROXIMATION FOR TUNNELING THROUGH NONUNIFORM BARRIERS}

We consider a lateral junction between two conductors separated by an insulating barrier of width $2 d$ (see Fig. 2) modeled by a potential of the form

$$
\mathcal{U}(x, \boldsymbol{\rho})=U_{0}+\sum_{i=1}^{N}\left[U\left(x,\left|\boldsymbol{\rho}-\boldsymbol{\rho}_{i}\right|\right)-U\left(x,\left|\boldsymbol{\rho}-\boldsymbol{\rho}_{i}^{\prime}\right|\right)\right]
$$

for $|x| \leq d$. The barrier inhomogeneity is described by the second term as the superposition of $N$ pairs of the oppositesign potentials centered at points $\boldsymbol{\rho}_{i}$ and $\boldsymbol{\rho}_{i}^{\prime}$, randomly distributed over a large junction area $A$. We do not distinguish the $x$ coordinates of the defects assuming $U(x,|\boldsymbol{\rho}|)$ to vary smoothly with $x$ across the barrier. The inhomogeneous part vanishes upon averaging over the junction area so that it represents the lateral spatial fluctuation of the barrier potential around a mean value $U_{0}$ (measured from the Fermi level $E_{F}$ ). While capturing generic features of randomly transparent barriers, this model, in particular, describes electrostatic disorder in an overall neutral insulator containing an equal amount of donors and acceptors. If they are distributed homogeneously, the Fermi level remains in the middle of the band gap $^{28}$ so that at sufficiently low bias voltage and temperature, we can neglect resonant tunneling. In the situations where resonant tunneling does contribute to the TMR, ${ }^{21,29}$ our model can still be used to qualitatively study the back- ground (nonresonant) contribution to the magnetoresistance as a function of the defect concentration in the barrier.

Within linear-response theory, ${ }^{30,31}$ we can express the junction conductance $g_{\sigma}$ (measured in units of $e^{2} / h$ ) for electrons with spin $\sigma=\uparrow, \downarrow$ at zero temperature as

$$
g_{\sigma}=(2 \pi \hbar)^{2} \sum_{k_{1} k_{2}}\left|J_{k_{1} k_{2}}\right|^{2} \delta\left(E_{F}-E_{\sigma k_{1}}\right) \delta\left(E_{F}-E_{\sigma k_{2}}\right) .
$$

The matrix elements of the current operator $J_{k_{1} \boldsymbol{k}_{2}}$ evaluated in the barrier separating conductors 1 and 2 describe spinconserving tunneling between their states with wave vectors $\boldsymbol{k}_{1}$ and $\boldsymbol{k}_{2}$ at energies $E_{\sigma k_{1}}$ and $E_{\sigma \boldsymbol{k}_{2}}$. The dependence of $J_{\boldsymbol{k}_{1} \boldsymbol{k}_{2}}$ on the components $k_{j x}$ perpendicular and $\boldsymbol{k}_{j \|}$ parallel to the interface $(j=1,2)$ is given by ${ }^{32-34}$

$$
\begin{gathered}
J_{\boldsymbol{k}_{1} \boldsymbol{k}_{2}}=\frac{i \hbar}{2 m_{B}} \psi_{k_{1 x}}(d) \psi_{k_{2 x}}(-d) W_{\boldsymbol{k}_{1 \|} \boldsymbol{k}_{2 \|},}, \\
W_{\boldsymbol{k}_{1||} \boldsymbol{k}_{2 \|}}=A^{-1} \int d \boldsymbol{\rho} \mathrm{e}^{i\left(\boldsymbol{k}_{2 \|}-\boldsymbol{k}_{1 \|}\right) \boldsymbol{\rho}} W(\boldsymbol{\rho}), \\
W(\boldsymbol{\rho})=\phi_{2}(x, \boldsymbol{\rho}) \partial_{x} \phi_{1}(x, \boldsymbol{\rho})-\phi_{1}(x, \boldsymbol{\rho}) \partial_{x} \phi_{2}(x, \boldsymbol{\rho}) .
\end{gathered}
$$

In Eq. (3), $m_{B}$ is the effective mass in the barrier. It is assumed that in the absence of tunneling, the one-particle states in the leads are $\psi_{k_{j x}}(x) \mathrm{e}^{i \boldsymbol{k}_{j \mid \boldsymbol{\rho}} \boldsymbol{\rho} / A^{1 / 2}}$, where the basis functions $\psi_{k_{j x}}(x)$ in the $x$ direction will be specified later. The tunneling coupling is accounted for by the Wronskian, Eq. (5), of the two independent solutions $\phi_{1,2}(x, \boldsymbol{\rho})$ of the Schrödinger equation inside the barrier, such that $\phi_{j}(x, \boldsymbol{\rho})$ decays into the barrier from side $j=1,2 .{ }^{32-34}$

To calculate $\phi_{1,2}(x, \boldsymbol{\rho})$, we assume that the inverse penetration length at the mean value of the barrier potential $\kappa$ $=\left(2 m_{B} U_{0} / \hbar^{2}\right)^{1 / 2}$ satisfies the conditions

$$
\kappa \gg \sqrt{2 m_{B} U(x, \boldsymbol{\rho})} / \hbar, \quad \kappa \gg k_{j \sigma},
$$

where $k_{j \sigma}$ are the Fermi wave vectors in the leads $j=1,2$. In this case, the tunneling problem becomes effectively one dimensional, which, together with the smoothness of $U(x, \boldsymbol{\rho})$ on the scale of $\kappa^{-1}$, allows us to employ the Glauber (eikonal) approximation frequently used in high-energy scattering theory. ${ }^{35}$ Under conditions (6), the barrier wave functions are

$$
\begin{aligned}
\phi_{j}(x, \boldsymbol{\rho}) \approx & \exp \{\kappa( \pm x-d) \\
& \left. \pm \sum_{i=1}^{N}\left[u_{ \pm}\left(x, \boldsymbol{\rho}-\boldsymbol{\rho}_{i}\right)-u_{ \pm}\left(x, \boldsymbol{\rho}-\boldsymbol{\rho}_{i}^{\prime}\right)\right]\right\}, \\
u_{ \pm}(x, \boldsymbol{\rho})= & \frac{\kappa}{2 U_{0}} \int_{ \pm d}^{x} d x^{\prime} U\left(x^{\prime},|\boldsymbol{\rho}|\right), \quad \kappa d \gg 1,
\end{aligned}
$$

where \pm correspond to the functions penetrating the barrier from sides $j=1,2$, respectively. By using Eqs. (7) and (8), we obtain for the Wronskian (5)

$$
W(\boldsymbol{\rho}) \approx W_{0} \exp \left\{\sum_{i=1}^{N}\left[u\left(\boldsymbol{\rho}-\boldsymbol{\rho}_{i}^{\prime}\right)-u\left(\boldsymbol{\rho}-\boldsymbol{\rho}_{i}\right)\right]\right\},
$$




$$
u(\boldsymbol{\rho})=\frac{\kappa}{2 U_{0}} \int_{-d}^{d} d x U(x,|\boldsymbol{\rho}|) .
$$

Here, $W_{0}=2 \kappa \exp (-2 \kappa d)$ is the Wronskian for a uniform barrier where the matrix (4) is proportional to a Kronecker delta $\delta_{\boldsymbol{k}_{1 \|} \boldsymbol{k}_{2 \|}}$. Equation (9) represents the main result of this section: In the tunneling overlap of the barrier wave functions, the lateral fluctuation of the potential is exponentially amplified and is not necessarily weak since $u$ [Eq. (10)] contains the large parameter $\kappa d$.

Equation (2) for the conductance can be recast into the more convenient form

$$
g_{\sigma}=\left(\frac{\pi \hbar^{2}}{m_{B}}\right)^{2} \sum_{k_{1 \|} k_{2 \|}}\left|W_{k_{1 \|} \boldsymbol{k}_{2 \|}}\right|^{2} \nu_{\sigma k_{1 \|}}(d) \nu_{\sigma \boldsymbol{k}_{2 \|}}(-d),
$$

where

$$
\nu_{\sigma k_{j \|}}( \pm d)=\sum_{k_{j x}}\left|\psi_{k_{j x}}( \pm d)\right|^{2} \delta\left(E_{F}-E_{\sigma k_{j}}\right)
$$

are the local densities of states for given $\boldsymbol{k}_{j \|}$ and $\sigma$ at the boundaries of systems 1 and 2 . We now make use of the explicit expressions

$$
\begin{gathered}
\psi_{k_{1 x}}(x)=\left(2 / L_{1}\right)^{1 / 2} \sin \left[k_{1 x}(x-d)+\gamma_{1}\right], \\
\psi_{k_{2 x}}(x)=\left(2 / L_{2}\right)^{1 / 2} \sin \left[k_{2 x}(x+d)-\gamma_{2}\right]
\end{gathered}
$$

for the basis functions in the $x$ direction, where $L_{1,2}$ are the lengths of the systems. The phases $\gamma_{1,2}=\arctan \left(k_{1,2 x} / \kappa\right)$ are determined by the boundary conditions $\partial_{x} \psi_{k_{1 x}}(d)=\kappa \psi_{k_{1 x}}(d)$ and $\partial_{x} \psi_{k_{2 x}}(-d)=-\kappa \psi_{k_{2 x}}(-d)$, valid for abrupt interfaces. ${ }^{38}$ Assuming, furthermore, spherical Fermi surfaces with effective mass $m$, we find

$$
\nu_{\sigma k_{j \|}}( \pm d)=\frac{2 m}{\pi \hbar^{2}} \frac{\left(k_{j \sigma}^{2}-k_{j \|}^{2}\right)^{1 / 2} \Theta\left(k_{j \sigma}-k_{j \|}\right)}{k_{j \sigma}^{2}+\kappa^{2}-k_{j \|}^{2}} .
$$

To finally prepare Eq. (11) for averaging over the ensemble of disorder realizations, we recast the square of the matrix elements $W_{\boldsymbol{k}_{1 \|} \boldsymbol{k}_{2 \|}}$ [Eq. (4)] as follows:

$$
\begin{aligned}
\left|W_{\boldsymbol{k}_{1 \|} \boldsymbol{k}_{2 \|}}\right|^{2} & =\int \frac{d \boldsymbol{\delta}}{A} e^{i\left(\boldsymbol{k}_{2 \|} \boldsymbol{k}_{1 \|}\right) \boldsymbol{\delta}} \times \int \frac{d \boldsymbol{\rho}}{A} W\left(\boldsymbol{\rho}+\frac{\boldsymbol{\delta}}{2}\right) W\left(\boldsymbol{\rho}-\frac{\boldsymbol{\delta}}{2}\right) \\
& \rightarrow \int \frac{d \boldsymbol{\delta}}{A} e^{i\left(\boldsymbol{k}_{2 \|}-\boldsymbol{k}_{1 \|}\right) \boldsymbol{\delta}}\left(W\left(\boldsymbol{\rho}+\frac{\boldsymbol{\delta}}{2}\right) W\left(\boldsymbol{\rho}-\frac{\boldsymbol{\delta}}{2}\right)\right\rangle_{\mathrm{conf}} .
\end{aligned}
$$

Here, averaging over different points, $\boldsymbol{\rho}$ on area $A$ is replaced by configurational averaging over uniformly distributed defect positions $\boldsymbol{\rho}_{i}$ and $\boldsymbol{\rho}_{i}^{\prime}$.

\section{CONFIGURATIONAL AVERAGING AND SPIN-DEPENDENT CONDUCTANCE}

\section{A. Averaging procedure}

To evaluate the correlation function of the Wronskian in Eq. (16) for large $N$ and $A$, we employ the HoltsmarkMarkov averaging procedure ${ }^{36}$ implemented as follows:

$$
\begin{aligned}
\left\langle W\left(\boldsymbol{\rho}_{1}\right) W\left(\boldsymbol{\rho}_{2}\right)\right\rangle & =W_{0}^{2}\left[\int \frac{d \boldsymbol{\rho}^{\prime}}{A} e^{u\left(\boldsymbol{\rho}_{1}-\boldsymbol{\rho}^{\prime}\right)+u\left(\boldsymbol{\rho}_{2}-\boldsymbol{\rho}^{\prime}\right)}\right]^{N}\left[\int \frac{d \boldsymbol{\rho}}{A} e^{-u\left(\boldsymbol{\rho}_{1}-\boldsymbol{\rho}\right)-u\left(\boldsymbol{\rho}_{2}-\boldsymbol{\rho}\right)}\right]^{N} \\
& =W_{0}^{2}\left\{1-\frac{n}{N} \int d \boldsymbol{\rho}^{\prime}\left[1-e^{u\left(\boldsymbol{\rho}_{1}-\boldsymbol{\rho}^{\prime}\right)+u\left(\boldsymbol{\rho}_{1}-\boldsymbol{\rho}^{\prime}\right)}\right]\right\}^{N}\left\{1-\frac{n}{N} \int d \boldsymbol{\rho}\left[1-e^{-u\left(\boldsymbol{\rho}_{1}-\boldsymbol{\rho}\right)-u\left(\boldsymbol{\rho}_{2}-\boldsymbol{\rho}\right)}\right]\right\}^{N} \\
& \approx W_{0}^{2} \exp \left\{-n \int d \boldsymbol{\rho}^{\prime}\left[1-e^{u\left(\boldsymbol{\rho}_{1}-\boldsymbol{\rho}^{\prime}\right)+u\left(\boldsymbol{\rho}_{2}-\boldsymbol{\rho}^{\prime}\right)}\right]\right\} \exp \left\{-n \int d \boldsymbol{\rho}\left[1-e^{-u\left(\boldsymbol{\rho}_{1}-\boldsymbol{\rho}\right)-u\left(\boldsymbol{\rho}_{2}-\boldsymbol{\rho}\right)}\right]\right\}
\end{aligned}
$$

In the last step, we took the limit $N, A \rightarrow \infty$, introducing a finite defect concentration $n=N / A$. Clearly, the above correlation function becomes independent of the "center-of-mass" position $\left(\boldsymbol{\rho}_{1}+\boldsymbol{\rho}_{2}\right) / 2$ and can be rewritten as

$$
\left\langle W\left(\boldsymbol{\rho}_{1}\right) W\left(\boldsymbol{\rho}_{2}\right)\right\rangle=\left\langle W^{2}\right\rangle \exp \left\{n\left[C\left(\boldsymbol{\rho}_{1}-\boldsymbol{\rho}_{2}\right)-C(0)\right]\right\}
$$

with

$$
\left\langle W^{2}\right\rangle=W_{0}^{2} \exp \left\{8 \pi n \int \rho d \rho \sinh ^{2} u(\rho)\right\},
$$

$$
C(\boldsymbol{\delta})=2 \int d \boldsymbol{\rho} \cosh [u(\boldsymbol{\rho}+\boldsymbol{\delta} / 2)+u(\boldsymbol{\rho}-\boldsymbol{\delta} / 2)]
$$

where $\boldsymbol{\delta}=\boldsymbol{\rho}_{1}-\boldsymbol{\rho}_{2}$. For the matrix elements (16), we then obtain

$$
\left|W_{\boldsymbol{k}_{1 \|} \boldsymbol{k}_{2 \|}}\right|^{2}=\left\langle W^{2}\right\rangle \int \frac{d \boldsymbol{\delta}}{A} e^{-n[C(0)-C(\boldsymbol{\delta})]-i\left(\boldsymbol{k}_{1 \|}-\boldsymbol{k}_{2 \|}\right) \boldsymbol{\delta}} .
$$

Since the integration involves a rapidly oscillating function, we expand $C(\boldsymbol{\delta})$ in powers of $\boldsymbol{\delta}$, 


$$
C(\boldsymbol{\delta}) \approx C(0)-\boldsymbol{\delta}^{2} \int 2 \pi \rho d \rho(d u / d \rho)^{2} \cosh 2 u(\rho) .
$$

Then, the integration can be easily performed yielding

$$
\left|W_{\boldsymbol{k}_{1 \|} \boldsymbol{k}_{2 \|}}\right|^{2} \approx\left\langle W^{2}\right\rangle \frac{2 \pi \rho_{c}^{2}}{A} e^{-1 / 2\left(\boldsymbol{k}_{1 \|}-\boldsymbol{k}_{2 \|}\right)^{2} \rho_{c}^{2}},
$$

where the radius

$$
\rho_{c}=\left[4 \pi n \int \rho d \rho(d u / d \rho)^{2} \cosh 2 u(\rho)\right]^{-1 / 2}
$$

characterizes the spatial decay of the correlations:

$$
\left\langle W\left(\boldsymbol{\rho}_{1}\right) W\left(\boldsymbol{\rho}_{2}\right)\right\rangle \approx\left\langle W^{2}\right\rangle \exp \left[-\left(\boldsymbol{\rho}_{1}-\boldsymbol{\rho}_{2}\right)^{2} / 2 \rho_{c}^{2}\right] .
$$

Equation (11) for the conductance then finally reads

$$
\begin{aligned}
g_{\sigma}= & \left.\frac{\hbar^{2}}{2 m_{B}}\right)^{2} \frac{\left\langle W^{2}\right\rangle A \rho_{c}^{2}}{2 \pi} \\
& \times \int d \boldsymbol{k}_{1 \|} d \boldsymbol{k}_{2 \|} e^{-1 / 2\left(k_{1 \|}-\left.\boldsymbol{k}_{2 \|}\right|^{2} \rho_{c}^{2}\right.} \nu_{\sigma k_{1 \|}}(d) \nu_{\sigma k_{2 \|}}(-d) .
\end{aligned}
$$

The effect of the disorder depends on the dimensionless parameters $\rho_{c} k_{1 \sigma}$ and $\rho_{c} k_{2 \sigma}$, controlling the crossover between the $\boldsymbol{k}_{\|}$-conserving and random tunneling regimes.

\section{B. $\boldsymbol{k}_{\|}$-conserving tunneling}

For weak disorder $\left(\rho_{c} k_{1,2 \sigma} \gg 1\right)$, the matrix elements in Eq. (25) have a sharp maximum at $\boldsymbol{k}_{1 \|}=\boldsymbol{k}_{2 \|}$ and hence can be integrated out. This yields the Landauer-type formula

$$
\begin{gathered}
g_{\sigma}=\frac{A}{(2 \pi)^{2}} \int d \boldsymbol{k}_{\|} T_{\sigma}\left(\boldsymbol{k}_{\|}\right), \\
T_{\sigma}\left(\boldsymbol{k}_{\|}\right)=\left(\frac{\pi W_{0} \hbar^{2}}{m}\right)^{2} \nu_{\sigma k_{\|}}(d) \nu_{\sigma k_{\|}}(-d),
\end{gathered}
$$

with $W_{0}$ from Eq. (9) and $T_{\sigma}\left(\boldsymbol{k}_{\|}\right)$being the transmission probability for a uniform rectangular barrier.

\section{Random-momentum tunneling}

This regime is reached in the limit $\rho_{c} k_{1,2 \sigma} \ll 1$ when the matrix elements become momentum independent. Then, the integrations over $\boldsymbol{k}_{1 \|}$ and $\boldsymbol{k}_{2 \|}$ can be done separately, yielding the conductance as the product of the local densities of states (DOS), $\nu_{\sigma}( \pm d)=\int d \boldsymbol{k}_{\|} \nu_{\sigma \boldsymbol{k}_{\|}}( \pm d) /(2 \pi)^{2}$,

$$
\begin{aligned}
& g_{\sigma}=(2 \pi)^{3}\left(\frac{\hbar^{2}}{2 m_{B}}\right)^{2}\left\langle W^{2}\right\rangle A \rho_{c}^{2} \nu_{\sigma}(d) \nu_{\sigma}(-d), \\
& \nu_{\sigma}( \pm d)=\nu_{j \sigma}^{\text {(bulk })}\left[1-\left(\kappa / k_{j \sigma}\right) \arctan \left(k_{j \sigma} / \kappa\right)\right] .
\end{aligned}
$$

The presence of the local DOS in Eq. (28) reflects the sharpness of the interfaces at $x= \pm d$. The difference between the local DOS [Eq. (29)] and the corresponding DOS in the bulk

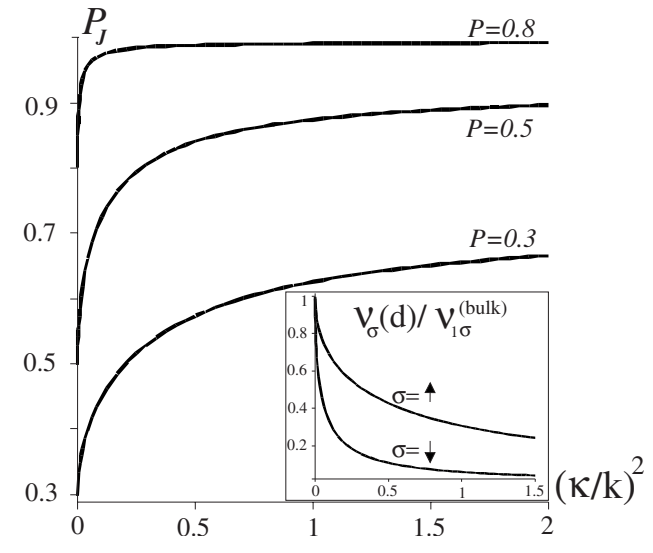

FIG. 3. Current spin polarization, Eq. (31), as a function of $(\kappa / k)^{2}$ characterizing the strength of the mean barrier potential $U_{0}$ for different values of the electron polarization, Eq. (32). Inset: Spin-resolved local DOS at the boundary of the spin injector versus $(\kappa / k)^{2}$ for $P=0.3 ; \kappa$ and $k$ are defined in text.

$\nu_{j \sigma}^{\text {(bulk) }}=m k_{j \sigma} / \pi^{2} \hbar^{2}$ is particularly pronounced for a high barrier with $k_{j \sigma} / \kappa \ll 1$ :

$$
\nu_{\sigma}( \pm d) \approx \nu_{j \sigma}^{\text {(bulk) }}\left(\frac{k_{j \sigma}}{\sqrt{3} \kappa}\right)^{2} \ll \nu_{j \sigma}^{(\text {bulk })}
$$

In the next section, we demonstrate that the randommomentum tunneling results in Jullière's magnetoresistance.

\section{TUNNELING SPIN POLARIZATION AND MAGNETORESISTANCE}

It is instructive to consider first the effect of the barrier disorder on the spin polarization of the tunneling current between a ferromagnet and a nonmagnetic conductor. For small bias voltages, the current spin polarization can be expressed in terms of the spin-resolved conductances:

$$
P_{J}=\left(g_{\uparrow}-g_{\downarrow}\right) /\left(g_{\uparrow}+g_{\downarrow}\right) .
$$

We assume that system 1 is a Stoner ferromagnet whose electron spin polarization $P$ is characterized by the bulk DOS for spin-up (majority) and spin-down (minority) carriers:

$$
P=\left(\nu_{1 \uparrow}^{\text {(bulk) }}-\nu_{1 \downarrow}^{\text {(bulk) })}\right) /\left(\nu_{1 \uparrow}^{\text {(bulk) }}+\nu_{1 \downarrow}^{\text {(bulk })}\right) .
$$

The Fermi wave vectors are parametrized as

$$
\begin{gathered}
k_{1 \uparrow}=k \sqrt{1+\Delta_{P}}, \quad k_{1 \downarrow}=k \sqrt{1-\Delta_{P}}, \\
\Delta_{P}=2 P /\left(1+P^{2}\right),
\end{gathered}
$$

where $\Delta_{P}$ is the dimensionless band spin-splitting and $k$ $=\sqrt{\left(k_{1 \uparrow}^{2}+k_{1 \uparrow}^{2}\right) / 2}$. System 2 has a spin-independent DOS $\nu_{2 \sigma}^{\text {(bulk) }}=\nu_{2}^{\text {(bulk) }}$ and Fermi wave vectors $k_{2 \sigma}=k$.

In the random tunneling regime, Eq. (28), the current spin polarization $P_{J}=\nu_{\uparrow}(d)-\nu_{\downarrow}(d) /\left(\nu_{\uparrow}(d)+\nu_{\downarrow}(d)\right)$ reflects the electron spin polarization at the surface of the spin injector, as shown in Fig. 3, $P_{J}$ increases with the ratio $(\kappa / k)^{2}$, characterizing the strength $U_{0}$ of the mean barrier potential. Such an enhancement can be traced back to the behavior of the 


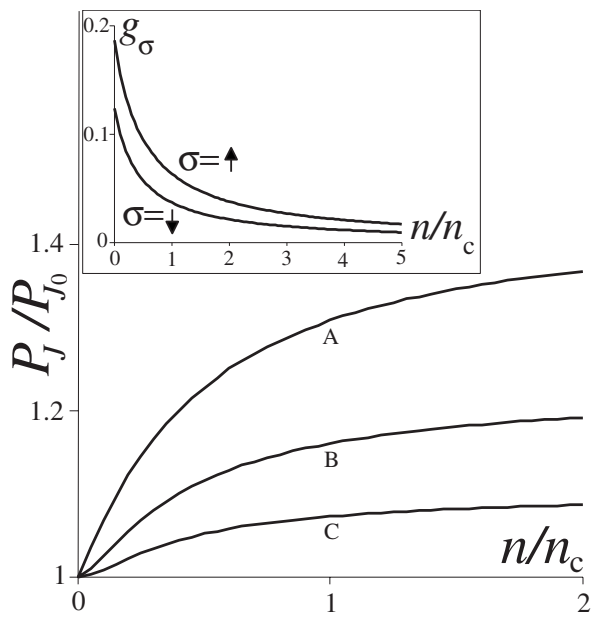

FIG. 4. Current spin polarization vs defect concentration in units of $P_{J_{0}}=P_{J}(n=0)$ and $n_{c}=n\left(\rho_{c} k\right)^{2}$ : (a) $P=0.1$, (b) $P=0.3$, and (c) $P=0.45$. Inset: spin-dependent conductances for $P=0.1$; for convenience, they are normalized to spin-independent factor $\left(m / m_{B}\right)^{2}\left\langle W^{2}\right\rangle A k^{2} / \kappa^{4}$.

spin-resolved local DOS (see inset in Fig. 3). Although both $\nu_{\uparrow}(d)$ and $\nu_{\downarrow}(d)$ are suppressed compared to the bulk values, the difference between them increases, leading to the higher surface spin polarization.

In what follows, we address exclusively systems with large values of $(\kappa / k)^{2}$. Then the double integral in Eq. (25) can be transformed to a single one in position representation: ${ }^{39}$

$$
\begin{aligned}
g_{\sigma}= & \frac{m^{2}}{m_{B}^{2}} \frac{\left(k_{1 \sigma} k_{2 \sigma}\right)^{3 / 2}}{\kappa^{4}}\left\langle W^{2}\right\rangle A \int_{0}^{\infty} d \delta \exp \left(-\frac{\delta^{2}}{2 \rho_{c}^{2}}\right) \\
& \times \frac{J_{3 / 2}\left(k_{1 \sigma} \delta\right) J_{3 / 2}\left(k_{2 \sigma} \delta\right)}{\delta^{2}},
\end{aligned}
$$

where $J_{3 / 2}(x)$ is a Bessel function. It follows from Eq. (35) that $P_{J}$ exceeds the clean-barrier value $P_{J_{0}}=P_{J}(n=0)$ at any finite defect concentration $n$ (see Fig. 4). There is a characteristic value $n_{c}$ related to the correlation radius, Eq. (23), as

$$
n_{c}=n\left(\rho_{c} k\right)^{2}
$$

The inset in Fig. 4 shows the corresponding behavior of the majority and minority electron conductances.

We wish to understand to what extent the behavior of the tunneling spin polarization $P_{J}$ correlates with that of the tunneling magnetoresistance. To this end, we consider a junction between two identical Stoner ferromagnets for which the TMR ratio is TMR $=1-\Sigma_{\sigma} g_{\sigma}^{A P} / \Sigma_{\sigma} g_{\sigma}^{P}$, where $g_{\sigma}^{P}$ and $g_{\sigma}^{A P}$ are both given by Eq. (35) with $k_{1 \sigma}$ and $k_{2 \sigma}$ defined in the following way. In the $P$ case, we choose the Fermi wave vectors of the majority and minority electrons to be $k_{\text {maj }}=k_{1 \uparrow}$ $=k_{2 \uparrow}$ and $k_{\text {min }}=k_{1 \downarrow}=k_{2 \downarrow}$, while for AP, $k_{\text {maj }}=k_{1 \uparrow}=k_{2 \downarrow}$ and $k_{\min }=k_{1}=k_{2 \uparrow}$. We again use the parametrization $k_{\text {maj }}$ $=k \sqrt{1+\Delta_{P}}$ and $k_{\min }=k \sqrt{1-\Delta_{P}}$ with $k$ and $\Delta_{P}$ defined earlier in Eqs. (33) and (34).

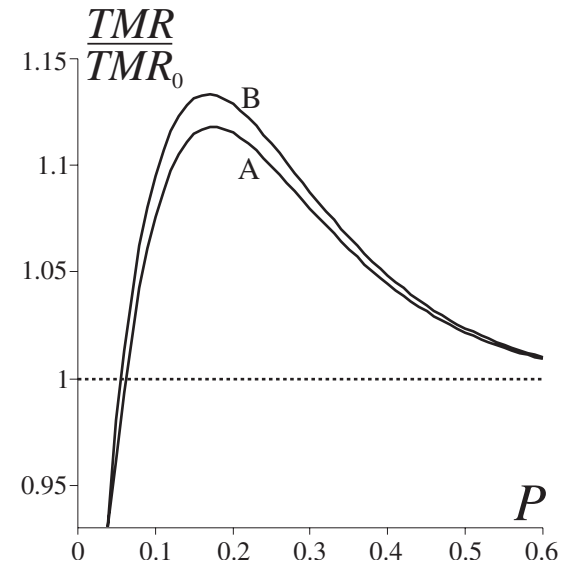

FIG. 5. Tunneling magnetoresistance as a function of bulk spin polarization for (a) $n=2.5 n_{c}$ and (b) $n=25 n_{c}$.

The dependence of the TMR on the defect concentration was already discussed in the introduction (see Fig. 1). Unlike $P_{J}(n)$, it is nonmonotonic with a minimum at $n \approx 0.25 n_{c}$ most pronounced for relatively low electron polarization (curves A-D). However, similar to $P_{J}(n)$, the TMR increases for $n / n_{c}>1$, saturating at the value

$$
\mathrm{TMR}=\left(\nu_{\text {maj }}-\nu_{\text {min }}\right)^{2} /\left(\nu_{\text {maj }}^{2}+\nu_{\text {min }}^{2}\right)
$$

depending solely on the local DOS of the majority and minority electrons $\nu_{\text {maj }}$ and $\nu_{\text {min }}$. Equation (37) follows from the asymptotic expression (28) and definitions of $k_{\text {maj }}$ and $k_{\min }$, reproducing the Jullière's result for a sharp barrier.

Another surprising feature of the TMR is that for strongly disordered barriers with $n / n_{c}>1$, the TMR ratio turns out to be a nonmonotonic function of electron spin polarization $P$, as shown in Fig. 5. By choosing the ferromagnets with $P$ $\approx 0.15-0.2$, one can take advantage of the barrier disorder to enhance the TMR response.

In conclusion, we discuss the applicability of our results. They are valid for the homogeneous defect distribution in a large-area barrier so that both the current spin polarization and TMR are independent of the junction cross-sectional geometry. They also do not depend on a concrete form of the defect potential $U(x, \boldsymbol{\rho})$, provided that the eikonal approximation holds (see Sec. II). Let us estimate the correlation radius $\rho_{c}$ [Eq. (23)] and the characteristic concentration $n_{c}$ [Eq. (36)] for a system of compensating donors and acceptors with charges $\pm e$ distributed on a plane $x=0$ in the middle of the barrier (so-called $\delta$ doping ${ }^{37}$ ). Since the defects release no charge carriers, we consider the screening of the defect potential $U(x, \boldsymbol{\rho})$ by the conducting electrodes. To simplify the calculations, we assume ideally screening electrodes described by the boundary conditions $U(x= \pm d, \boldsymbol{\rho})$ $=0$ for the Poisson equation $\Delta U=\left(4 \pi e^{2} / \epsilon\right) \delta(x) \delta(\boldsymbol{\rho})$, where $\epsilon$ is the dielectric constant of the barrier material. The solution of such a boundary problem is a superposition of the Coulomb potentials of the defect charge and the image ones,

$$
U(x, \boldsymbol{\rho})=\frac{e^{2}}{\epsilon} \sum_{n=-\infty}^{\infty} \frac{(-1)^{n}}{\sqrt{(x-2 d n)^{2}+\boldsymbol{\rho}^{2}}},
$$

where terms with $n= \pm 1, \pm 2, \ldots$ represent an infinite signalternating series of image charges at points $x=2 d n$, gener- 
ated by multiple "reflections" in two "mirrors" $x= \pm d$. The boundary conditions are satisfied due to the even and odd terms canceling each other at $x= \pm d$. For the estimate, it suffice to know the asymptotic formula for $\rho \gg d$, obtained by replacing $\Sigma_{n} \rightarrow \int d n$ and $(-1)^{n} \rightarrow \cos (\pi n)$ in Eq. (38). The integration yields

$$
U(x, \boldsymbol{\rho}) \approx \frac{e^{2}}{d \epsilon} \cos \left(\frac{\pi x}{2 d}\right) K_{0}\left(\frac{\pi|\boldsymbol{\rho}|}{2 d}\right),
$$

where $K_{0}(x)$ is the modified Bessel function of second kind.

It can be checked numerically that Eq. (39) is also a fairly good approximation for $\rho \leq d$, except for the immediate vicinity of the charge location $x=0, \rho=0$. As for our system $\kappa d \gg 1$ [see, Eq. (8)], the $x$ dependence of the potential, Eq. (39), is smooth on the scale of the electronic penetration length $\kappa^{-1}$. This justifies our quasiclassical approach, in particular, allowing us to neglect smearing of the defect distribution about the plane $x=0$ as long as they are still deep inside the barrier. For experimental situations where charged defects occur at the barrier boundaries or inside the conductors, our model needs to be modified to account for the Thomas-Fermi screening and scattering from such defects.

By using Eqs. (23) and (39), we find the correlation radius

$$
\rho_{c}=\frac{\kappa a_{B}}{8\left[\pi n \int_{0}^{\infty} \xi d \xi\left(\frac{d K_{0}(\xi)}{d \xi}\right)^{2} \cosh \left(\frac{8 K_{0}(\xi)}{\pi \kappa a_{B}}\right)\right]^{1 / 2}},
$$

where $a_{B}=\epsilon \hbar^{2} / e^{2} m_{B}$ is the effective Bohr radius. It must satisfy the condition of a weak potential fluctuation $\kappa a_{B} \gg 1$, equivalent to the first inequality in Eq. (6). For instance, if we take $\kappa a_{B}=8$, then $\rho_{c} \approx n^{-1 / 2}$ up to a numerical factor, i.e., the correlation radius is roughly the average distance between the defects. Consequently, the characteristic defect concentration $n_{c}$ at which the TMR approaches Jullière's limit is related to the Fermi wave vector $k$ as $n_{c} \approx k^{2}$. This condition is easier to meet for magnetic semiconductors than for ferromagnetic transition metals. As we saw, however, the long-range barrier disorder can affect the TMR at significantly lower concentrations.

\section{ACKNOWLEDGMENTS}

We thank J. Fabian, D. Ryndyk, D. Weiss, and M. Wimmer for stimulating discussions. The work was supported by the Deutsche Forschungsgemeinschaft within SFB 689.
${ }^{1}$ J. S. Moodera, L. R. Kinder, T. M. Wong, and R. Meservey, Phys. Rev. Lett. 74, 3273 (1995).

${ }^{2}$ T. Miyazaki and N. Tezuka, J. Magn. Magn. Mater. 139, L231 (1995).

${ }^{3}$ S. Parkin, C. Kaiser, A. Panchula, P. Rice, B. Hughes, M. Samant, and S.-H. Yang, Nat. Mater. 3, 862 (2004).

${ }^{4}$ I. Zutić, J. Fabian, and S. Das Sarma, Rev. Mod. Phys. 76, 323 (2004).

${ }^{5}$ J. C. Slonczewski, Phys. Rev. B 39, 6995 (1989).

${ }^{6}$ A. M. Bratkovsky, Phys. Rev. B 56, 2344 (1997).

${ }^{7}$ J. M. MacLaren, X.-G. Zhang, and W. H. Butler, Phys. Rev. B 56, 11827 (1997).

${ }^{8}$ P. Mavropoulos, N. Papanikolaou, and P. H. Dederichs, Phys. Rev. Lett. 85, 1088 (2000).

${ }^{9}$ J. Mathon and A. Umerski, Phys. Rev. B 63, 220403(R) (2001).

${ }^{10}$ W. H. Butler, X.-G. Zhang, T. C. Schulthess, and J. M. MacLaren, Phys. Rev. B 63, 054416 (2001).

${ }^{11}$ N. N. Beletskii, G. P. Berman, A. R. Bishop, S. A. Borysenko, and V. M. Yakovenko, Phys. Rev. B 75, 174418 (2007).

${ }^{12}$ C. Heiliger, M. Gradhand, P. Zahn, and I. Mertig, Phys. Rev. Lett. 99, 066804 (2007).

${ }^{13}$ S. Zhang, P. M. Levy, A. C. Marley, and S. S. P. Parkin, Phys. Rev. Lett. 79, 3744 (1997).

${ }^{14}$ A. H. MacDonald, T. Jungwirth, and M. Kasner, Phys. Rev. Lett. 81, 705 (1998).

${ }^{15}$ F. Guinea, Phys. Rev. B 58, 9212 (1998).

${ }^{16}$ A. M. Bratkovsky, Appl. Phys. Lett. 72, 2334 (1998).

${ }^{17}$ J. Hong, R. Q. Wu, and D. L. Mills, Phys. Rev. B 66, 100406(R) (2002).

${ }^{18}$ G. Tkachov, E. McCann, and V. I. Fal'ko, Phys. Rev. B 65, 024519 (2001); Also in Recent Progress in Many-Body Theories, edited by R. F. Bishop, T. Brands, K. A. Gernoth, N. R.
Walet, and Y. Xian (World Scientific, Singapore, 2002), Vol. 6, p. 60 .

${ }^{19}$ E. McCann, G. Tkachov, and V. I. Fal'ko, Physica E (Amsterdam) 12, 938 (2002).

${ }^{20}$ E. Yu. Tsymbal and D. G. Pettifor, Phys. Rev. B 58, 432 (1998).

${ }^{21}$ E. Y. Tsymbal, O. Mryasov, and P. R. LeClair, J. Phys.: Condens. Matter 15, R109 (2003).

${ }^{22}$ J. Mathon and A. Umerski, Phys. Rev. B 74, 140404(R) (2006).

${ }^{23}$ P. X. Xu, V. M. Karpan, K. Xia, M. Zwierzycki, I. Marushchenko, and P. J. Kelly, Phys. Rev. B 73, 180402(R) (2006).

${ }^{24}$ A. Vedyayev, D. Bagrets, A. Bagrets, and B. Dieny, Phys. Rev. B 63, 064429 (2001).

${ }^{25}$ M. Jullière, Phys. Lett. 54A, 225 (1975).

${ }^{26}$ S. Kreuzer, J. Moser, W. Wegscheider, D. Weiss, M. Bichler, and D. Schuh, Appl. Phys. Lett. 80, 4582 (2002).

${ }^{27}$ M. Zenger, J. Moser, W. Wegscheider, D. Weiss, and T. Dietl, J. Appl. Phys. 96, 2400 (2004).

${ }^{28}$ See, e.g., B. I. Shklovskii and A. L. Efros, Electronic Properties of Doped Semiconductors (Springer, Berlin, 1984).

${ }^{29}$ J. Mathon and A. Umerski, Phys. Rev. B 71, 220402(R) (2005).

${ }^{30}$ R. Kubo, J. Phys. Soc. Jpn. 12, 570 (1957).

${ }^{31}$ D. A. Greenwood, Proc. Phys. Soc. London 71, 585 (1958).

${ }^{32}$ J. Bardeen, Phys. Rev. Lett. 6, 57 (1961).

${ }^{33}$ W. A. Harrison, Phys. Rev. 123, 85 (1961).

${ }^{34}$ C. B. Duke, in Tunneling in Solids, Solid State Physics Suppl. 10 (Academic, New York, 1969).

${ }^{35}$ R. J. Glauber, Phys. Rev. 100, 242 (1955).

${ }^{36}$ See, e.g., S. Chandrasekhar, Rev. Mod. Phys. 15, 1 (1943).

${ }^{37}$ R. Jansen and J. S. Moodera, Phys. Rev. B 61, 9047 (2000).

${ }^{38}$ Here, we ignore small corrections of order of $U(x,|\boldsymbol{\rho}|) / U_{0}$.

${ }^{39}$ This is a fairly good approximation for $(\kappa / k)^{2} \geq 5$. 